\title{
ARTICLE OPEN Scalable on-chip quantum state tomography
}

\author{
James G. Titchener ${ }^{1,2}$, Markus Gräfe ${ }^{2,3}$, René Heilmann ${ }^{2}$, Alexander S. Solntsev ${ }^{1,4}$, Alexander Szameit $\mathbb{I D}^{2,5}$ and Andrey A. Sukhorukov $\mathbb{D}^{1}$
}

Quantum information systems are on a path to vastly exceed the complexity of any classical device. The number of entangled qubits in quantum devices is rapidly increasing, and the information required to fully describe these systems scales exponentially with qubit number. This scaling is the key benefit of quantum systems, however it also presents a severe challenge. To characterize such systems typically requires an exponentially long sequence of different measurements, becoming highly resource demanding for large numbers of qubits. Here we propose and demonstrate a novel and scalable method for characterizing quantum systems based on expanding a multi-photon state to larger dimensionality. We establish that the complexity of this new measurement technique only scales linearly with the number of qubits, while providing a tomographically complete set of data without a need for reconfigurability. We experimentally demonstrate an integrated photonic chip capable of measuring two- and three-photon quantum states with statistical reconstruction fidelity of $99.71 \%$.

npj Quantum Information (2018)4:19; doi:10.1038/s41534-018-0063-5

\section{INTRODUCTION}

The standard way to characterize a quantum system ${ }^{1-6}$ is known as quantum state tomography., It involves measuring expectation values of a complete set of observables and using these to reconstruct the system's density matrix. ${ }^{8-13}$ To characterize an $\mathrm{N}$ qubit state, $2^{2 N}$ different observables are measured, ${ }^{8}$ thus the measurement apparatus must be reconfigured exponentially many times, which is impractical for large states. Furthermore many of the expectation values measured will be vanishingly small, and thus contribute little useful information. Finally, even if all the measurements can be completed, the task of reconstructing the density matrix from measurement data becomes computationally challenging for high qubit-number states. ${ }^{6}$

New approaches to quantum state tomography are being developed in an effort to increase its practicality and efficiency. Some approaches seek to avoid unnecessary measurements by assuming that the system is in particular low-rank states, such as sparse states ${ }^{14,15}$ or low dimensional matrix product states. ${ }^{16}$ Alternatively tomography can be "self-guided", where real-time feedback of measurement results guides the next choice of the measurement basis, ${ }^{17-19}$ helping to avoid taking measurements that have limited utility for analyzing the state. It has been shown that tomography procedures involving some global quantum measurements have increased error robustness relative to using only local qubit measurements, and thus can be completed in less time. ${ }^{20}$ The computational burden of inverting large data sets to find the density matrix is reduced with simple real-time optimization algorithms in self guided tomography, or can be completely avoided with systems for direct projection of density matrix parameters. ${ }^{21-23}$ However all these approaches rely on a common measurement paradigm, whereby different characteristics of a system are measured sequentially, thus they become exponentially complex to implement as the number of parameters in the density matrix scales exponentially with qubit number.

Here we present a quantum tomography method with experimental device complexity that scales linearly with qubit number. This is achieved by leveraging quantum systems' greatest strength, the simultaneous occupation of exponentially many states. Instead of preforming a sequence of different measurements on the state, we design a single static measurement system that preforms one, many-outcome measurement (Fig. 1a). It has been shown that multiplexing multiple on-off single photon detectors can allow photon-number resolved detection of quantum states of light. ${ }^{24}$ Here we demonstrate experimentally that, with the appropriate optical transformation, multiplexed detection can also allow full quantum state tomography, and we present a comprehensive theoretical analysis of multi-photon case building on our original proposal for two-photon tomography with static measurement setups ${ }^{25}$ (after the initial submission of this work, a similar approach to quantum state tomography was investigated theoretically in ref. ${ }^{26}$ ). When an $N$ photon state is spread coherently across an increased number of outputs, the number of possible $\mathrm{N}$-photon correlation measurement outcomes follows a similar scaling to the number of parameters in the density matrix. Thus the exponential scaling of quantum sates can be balanced by a similar scaling in the amount of information extracted from measurement of the state. This leads to the striking benefit that the required physical complexity of multi-outcome measurement only scales linearly with the number of qubits in the state being measured (Fig. 1b), in contrast to the usual exponential scaling.

In the context of photonic quantum states our approach also removes the need to build complex reconfigurable measurement systems, ${ }^{8}$ instead allowing full quantum tomography with just static linear optical circuits, which can easily be implemented on

\footnotetext{
${ }^{1}$ Nonlinear Physics Centre, Research School of Physics and Engineering, Australian National University, Canberra, ACT 2601, Australia; ${ }^{2}$ Institute of Applied Physics, Abbe Center of Photonics, Friedrich-Schiller-Universitt Jena, Max-Wien-Platz 1, Jena 07743, Germany; ${ }^{3}$ Fraunhofer Institute for Applied Optics and Precision Engineering, Albert-Einstein-Str. 7,

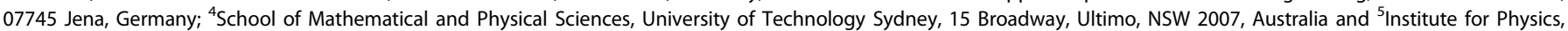
University of Rostock, Albert-Einstein-Strasse 23, 18059 Rostock, Germany

Correspondence: James G. Titchener (j.g.titchener@gmail.com)
}

Received: 16 May 2017 Revised: 10 January 2018 Accepted: 19 January 2018

Published online: 08 March 2018 
a

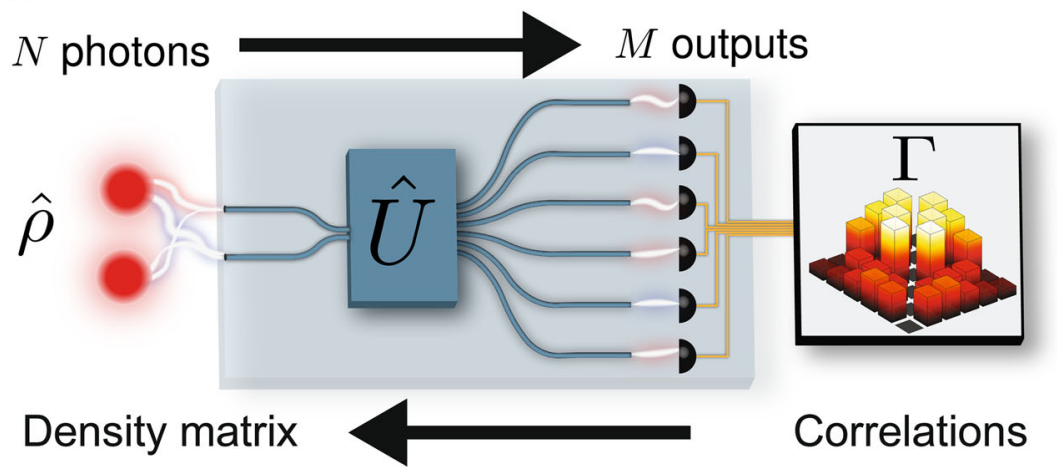

b

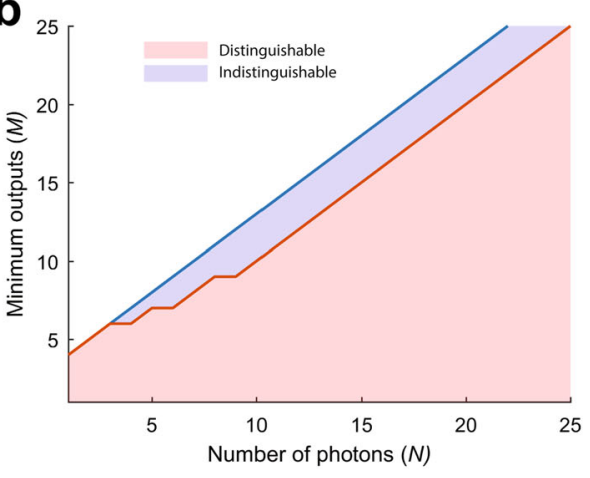

Fig. 1 Linearly scalable quantum state tomography concept. a Conceptual diagram of a photonic chip for scalable tomography of $N$-photon states based on a single linear transformation with two input modes and $M$ outputs. To enable reconstruction, the number of different $N$ photon correlations that can be measured at the output needs to be the same or larger than the number of independent parameters defining the input density matrix $\mathbf{b}$ Scaling relationship between the number of photons in the quantum state to be measured and the number of required output waveguides. The scaling for distinguishable and indistinguishable photon detection schemes is linear at large photon numbers, as indicated by red and blue lines, respectively (see Sec. I in Supplementary Material for details of derivation)

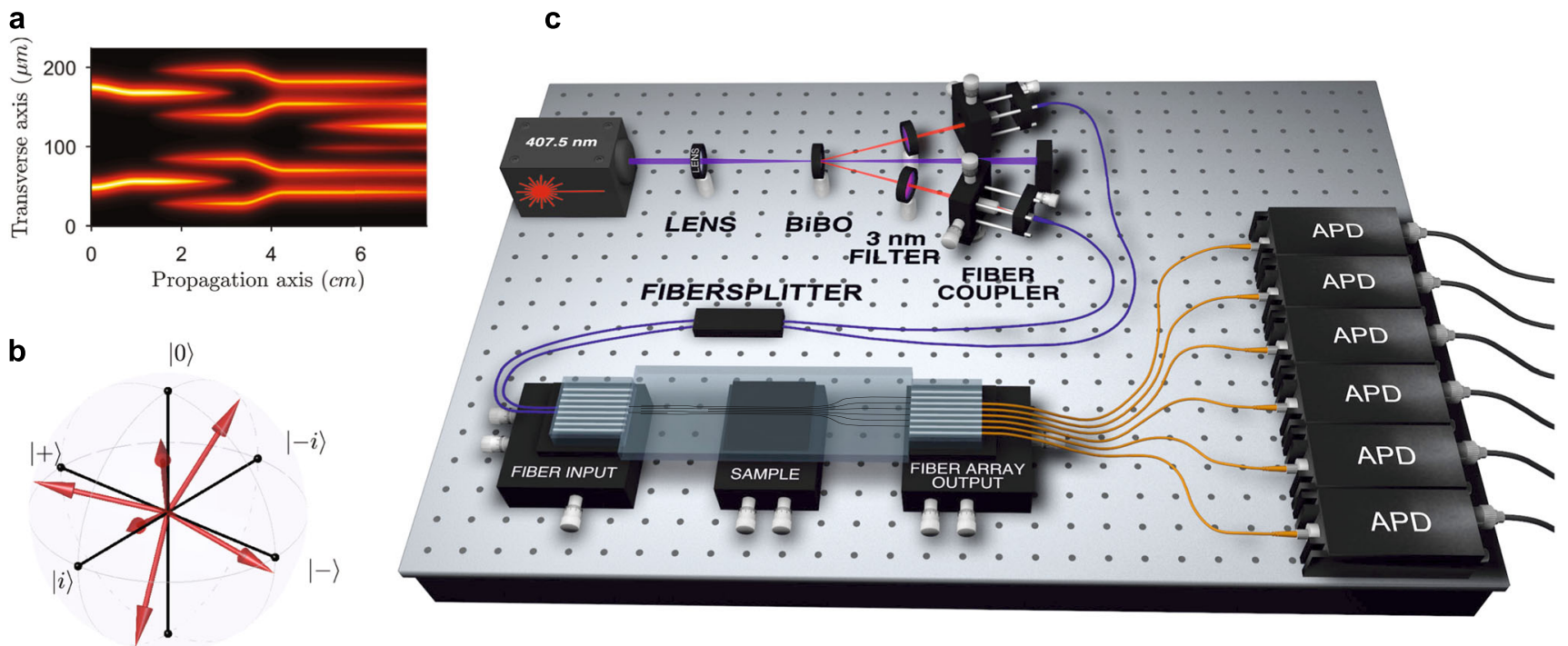

$|1\rangle$

Fig. 2 Experimental realization with a photonic chip. a The structure of the silica photonic chip showing simulation of coupling a single photon in state $|0\rangle+i|1\rangle$ into the input ports. b Experimentally determined mappings from the input Bloch sphere to the six output waveguide field intensities. c The experimental setup. Photon-pairs at $815 \mathrm{~nm}$ are generated via spontaneous parametric down-conversion (SPDC) by pumping a bismuth triborate $(\mathrm{BiBO})$ crystal with a 407.5-nm diode laser. The two photons are then coupled into two fibers, and optionally passed through a fiber-splitter to transform the anti-bunched state into a bunched state. The photons are then coupled to the photonic chip and detected with an array of six single photon-detecting avalanche photo-diodes (APD's)

photonic chips. This avoids the problem faced by conventional approaches, where some measurements provide little useful information about the underlying state. This is because in our approach, the full complement of measurements are performed simultaneously, and thus the most important correlation detections for reconstructing a particular state naturally have the highest count rates. Our approach is based on interfering all the photons through a special unitary transformation, thus can be optimized to incorporate nonlocal measurement, allowing the error robustness to be increased. Furthermore, we show that our approach is compatible with computationally scalable reconstruction, avoiding resource intensive direct inversion.

\section{RESULTS}

We explain our method for the case of $\mathrm{N}$-photons in an arbitrary pure or mixed state featuring spatial quantum entanglement between two input ports, although a larger number of ports can be considered as well. ${ }^{25}$ The $\mathrm{N}$-photon quantum state is described by a density matrix, $\hat{\rho}_{i n}$. We apply a single (fixed) linear transformation, $\hat{U}$, to map the quantum state from two inputs to a larger number of output waveguides, $M$, as illustrated in Fig. 1 a. Then, we obtain information about the quantum state by measuring coincidences, $\Gamma$, in the arrival time of photons to different combinations of the single-photon detectors. If the detectors can distinguish photons (i.e., by arrival time for time-bin encoded states), but cannot resolve photon numbers, then there are $M ! /(M-N)$ ! different $N$-photon correlations which can be measured, while the density matrix is described by $2^{2 N}$ real parameters. Then, its reconstruction is possible if the number of different correlations is larger than the number of unknown density matrix elements. We establish that the minimum number of output ports required scales linearly with the number of photons and approaches $M=N$ for large photon numbers, see red 

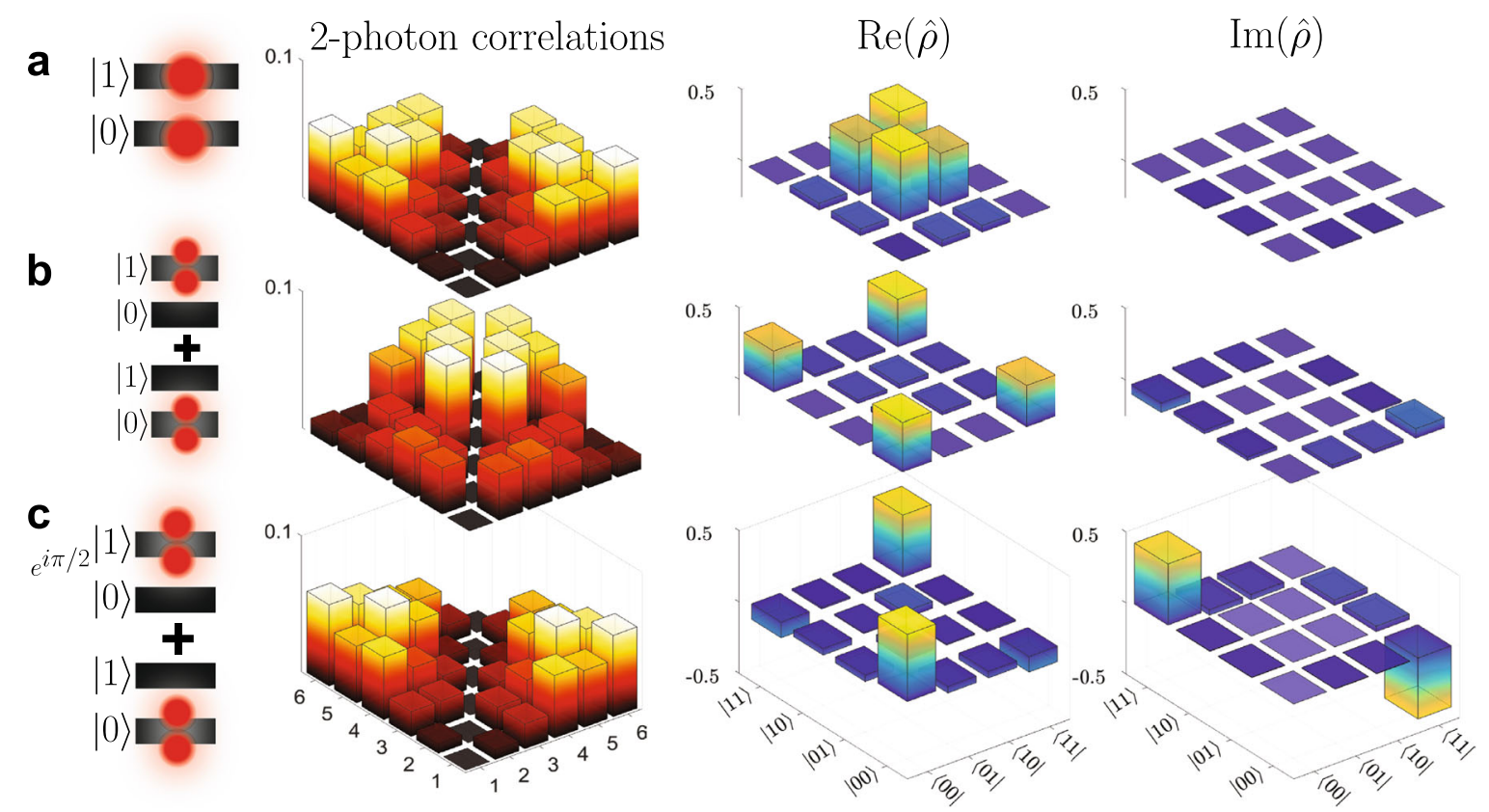

Fig. 3 Two photon tomography results. a Results for an anti-bunched state, $\mathbf{b}$ a N00N state, and $\mathbf{c}$ a N00N state with $\pi / 2$ phase shift. The second column shows the measured two-photon correlations, while the third and fourth columns show the real and imaginary parts of the recovered density matrices

line in Fig. 1b. In case of indistinguishable detection, when different photons produce the same kind of signal from the detectors, the reconstruction is possible for $M=N+3$ output ports, see blue line in Fig. 1b. We prove that linear scaling vs. photon numbers also holds for larger number of input ports, see detailed mathematical derivation in Sec. I of the Supplementary Material.

We experimentally demonstrate our approach by performing tomography of spatially entangled mixed or pure states of two indistinguishable photons, using a specially designed on-chip laser-written waveguide circuit. ${ }^{27}$ The action of the circuit on the input single photon state $|\psi\rangle=|0\rangle+i|1\rangle$ is shown in Fig. 2a. The circuit allows full reconstruction of the input density matrix just by measuring the output two-photon coincidences with non-photonnumber resolving single-photon detectors.

The circuit was optimized to make the tomographic reconstruction highly robust to measurement errors. Each output waveguide carries information from a different vector on the input Bloch sphere. The Bloch vectors were determined experimentally using a classical characterization method, ${ }^{28}$ and are shown graphically in Fig. 2b. The equal spacing of vectors around the Bloch sphere gives the device maximum robustness to errors in the tomography procedure, ${ }^{29}$ by essentially realizing non-local measurements. This can be confirmed by calculating the condition number of the transfer function of the chip, lower values of which correspond to higher robustness of the state reconstruction to measurement errors. The experimentally realized device has a condition number of $\simeq 5$, which is better than the condition number of $\simeq 9$ for typical tomography. ${ }^{20}$

To test the performance of the device we prepared a range of different two-photon entangled quantum states, coupled them into the chip, and measured the output correlations as schematically shown in Fig. 2c. We first analyze an anti-bunched state, which in an ideal form is described by the pure wavefunction $|\psi\rangle=(|01\rangle+|10\rangle) / \sqrt{2}$ and the corresponding density matrix $\hat{\rho}=|\psi\rangle\langle\psi|$. We present in Fig. 3a the experimentally measured probabilities of detecting the photon-pair in a given pair of output waveguides, and the reconstructed real and imaginary parts of the density matrix, as indicated by labels. We confirm that this is indeed an anti-bunched state, with the fidelity of $94.0 \%$. This value is actually limited by the quality of the photon source (which Hong-Ou-Mandel visibility is limited at 93\%), whereas we estimate the statistical reconstruction fidelity at the level of $99.71 \%$ (see Methods, Algorithmic reconstruction). Furthermore, this measurement permits us to get information about the spectral overlap of the pair of photons, since the observed correlations exhibit a generalized form of Hong-OuMandel interference, ${ }^{30-32}$ see Sec. III of the Supplementary Material for details.

We also prepared N0ON states, with wavefunctions given by $|\psi\rangle=\left(|00\rangle+e^{i \phi}|11\rangle\right) / \sqrt{2}$, where $\phi$ is a phase shift. The phase shift is determined by the photon propagation before the chip, and because the shift is double the value that would accumulate classically ${ }^{33}$ it is highly sensitive to the environment. Experimentally, we explored this quantum-enhanced sensitivity by propagating a two-photon NOON state through 1-m long optical fibers before the chip. The accumulated phase was very sensitive to fiber stress, varying by up to $2 \pi$ on the scale of a few minutes. With our approach we observed experimentally the temporal variation of the phase in the density matrix using an integration time of $20 \mathrm{~s}$ with a photon pair detection rate of $30 \mathrm{~Hz}$. We show typical twophoton correlations at different times in Fig. $2 b, c$ and the corresponding reconstructed NOON states, with phases determined to be $\phi \simeq 0$ in Fig. $3 \mathrm{~b}$ and $\phi \simeq \pi / 2$ in Fig. 3c. The fidelity of both states exceeds $94 \%$. Thus, we can observe with high precision the density matrix of a quantum state that is varying over time (a video of the time evolution of the density matrix is included in the Supplementary Material).

The reconstruction of the density matrices from the measured correlations was carried out using a computationally scalable algorithm. This is important, since although the number of detectors and waveguides in the circuit scales linearly with the number of qubits to be measured, the number of photon detections required for reconstruction still necessarily scales exponentially (as in standard quantum state tomography) and processing of measurement data would be extremely resource demanding for large photon numbers. We employ an optimization technique known as the simultaneous perturbation stochastic approximation (SPSA), ${ }^{34}$ similar to the algorithm formulated for 


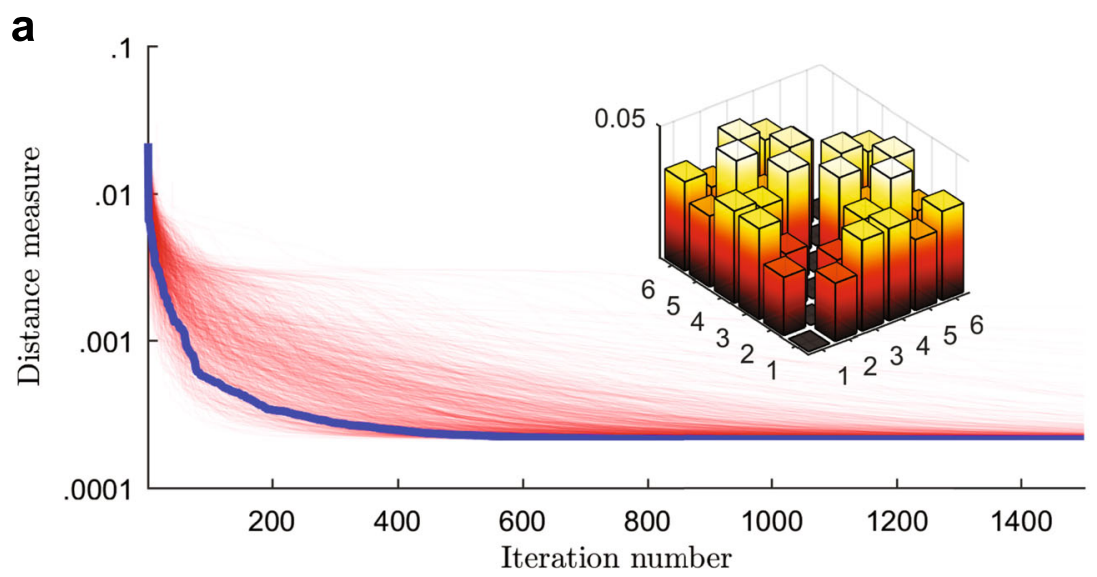

b
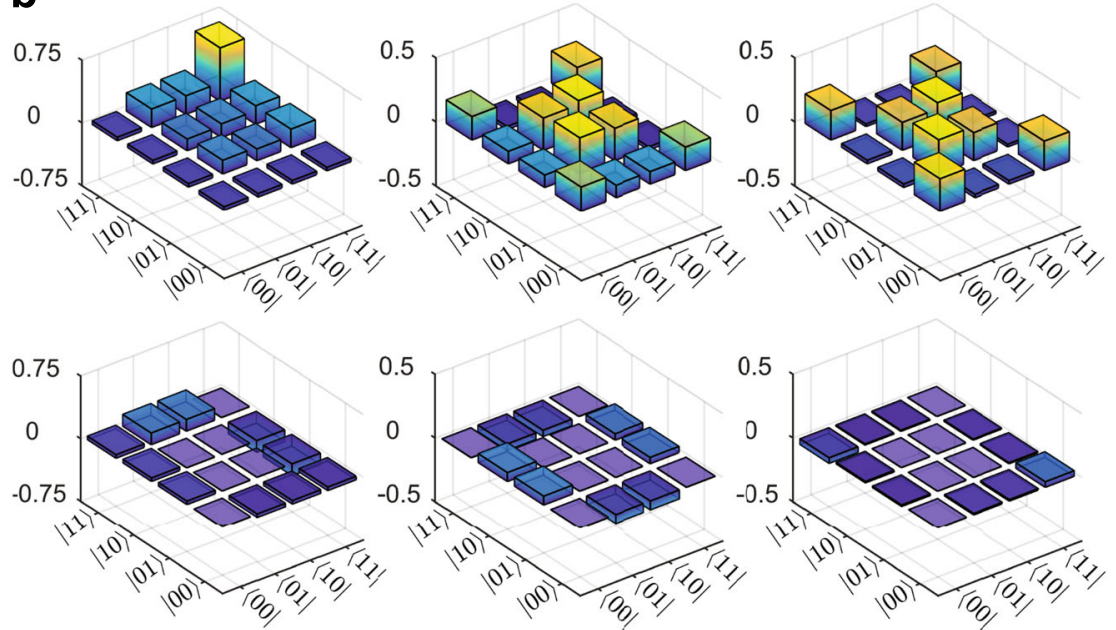

Fig. 4 Scalable reconstruction algorithm for mixed states. a (Inset) Measured two-photon correlations for the mixed state $\hat{\rho}_{\text {mix }}=\left(\hat{\rho}_{\text {anti-bunched }}+\hat{\rho}_{\text {NOON }}\right) / 2$. a Performance of the self guided tomography algorithm searching for the input density matrix that best matches the measured correlations. Red curves show 1000 different realizations of the algorithm. $\mathbf{b}$ The real (top row) and imaginary (bottom row) parts of the density matrix at iteration numbers 1, 30 and 300 during the realization highlighted with blue in Fig. $4 a$

self-guided quantum state tomography..$^{17}$ It minimizes a distance measure between the true state and the algorithm's current guess. We use the least squares distance between the measured correlations and the correlations that would be produced by the current guess of the density matrix, $\left|\Gamma_{\text {meas. }}-\Gamma_{\text {guess }}\right|^{2}$. The reconstruction fidelity of the algorithm is $99.67 \%$ after 1500 iterations (see Methods).

We demonstrate recovery of the mixed state $\hat{\rho}_{\text {mix }}=$ $\left(\hat{\rho}_{\text {anti-bunched }}+\hat{\rho}_{\text {NoON }}\right) / 2$ using the algorithm. The inset of Fig. $4 a$ shows the experimentally measured correlations corresponding to $\hat{\rho}_{\text {mix }}$, obtained by numerically combining separately recorded raw coincidence data from an anti-bunched state and a N0ON state. The main plot in Fig. 4a shows 1000 realizations of the algorithm as red lines, and density matrices at iteration numbers 1, 30 and 300 are shown in Fig. $4 \mathrm{~b}$ for the realization highlighted in blue in Fig. 4a. Importantly, our method offers the same computational advantage as self-guided tomography, but without a need for complex reconfigurable measurements which so far restricted this approach to only pure states. ${ }^{18}$

The chip presented in Fig. $2 a$ is also capable of tomography of degenerate three-photon states. We demonstrate this using the experimentally determined transfer function, $\hat{U}$, and simulate the propagation of a three-photon Greenberger-Horne-Zeilinger (GHZ) state (Fig. 5a) through the chip. The simulated output three-photon correlations are shown in Fig. 5b, where Gaussian noise with standard deviation $5 \%$ of the peak correlation value has been added to each element. Reconstruction of the input density matrix gives highly accurate results despite this noise. The real part of the recovered density matrix is shown in Fig. $5 \mathrm{~d}$, which closely matches the three-photon $\mathrm{GHZ}$ state in Fig. 5c. This provides a significantly simpler and more stable platform for three-photon tomography compared to previous realizations. ${ }^{35}$

\section{DISCUSSION}

We have demonstrated that a fixed linear optical transformation can be devised to allow complete quantum tomography of $\mathrm{N}$ photon states. Importantly, the complexity of the transformation only scales linearly with the number of photons in the state, in contrast to the exponential scaling in the number of measurements required in usual tomography. ${ }^{36}$ Due to its simplicity and lack of tunable elements, this approach to quantum measurement is uniquely suited to integration with on-chip super-conducting single-photon detectors ${ }^{37}$ for a fully on-chip tomography scheme. The combination of linear scaling in the required number of single photon detectors, as well as compatibility with integrated detection, suggests that the characterization of high photon number states is achievable. This provides a promising way to facilitate the characterization and development of increasingly complex quantum communication and computation systems. 
a

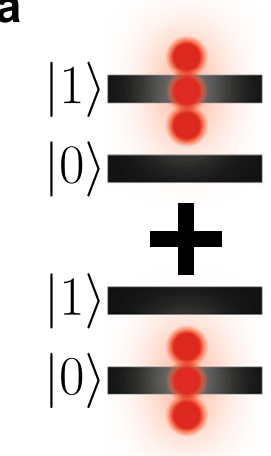

b

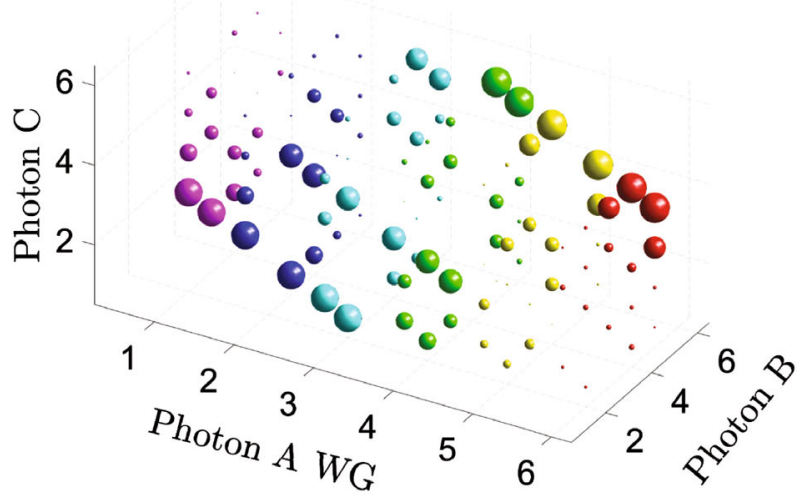

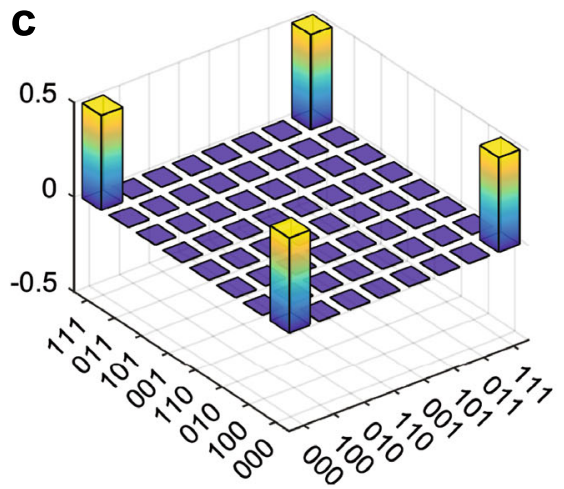

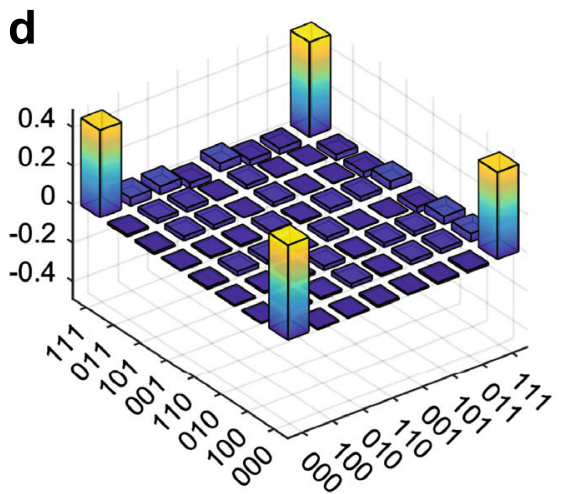

Fig. 5 Tomography of a three-photon state. a Diagram of the three-photon GHZ state. $\mathbf{b}$ Simulated correlations of the GHZ state after propagation through the measured transfer function of the device in Fig. 2a. Gaussian error with standard deviation of $5 \%$ the maximum correlation element's value is added to each element. c The real part of the GHZ state's density matrix. $\mathbf{d}$ Real part of the density matrix that was recovered using the simulated correlations from (b)

\section{METHODS}

Direct waveguide writing

We write our waveguides into transparent fused silica wafers (Corning 7980 ArF Grade), using ultrashort laser pulses $(\tau<150 \mathrm{fs}, \lambda=800 \mathrm{~nm})$ that are focused $250 \mu \mathrm{m}$ below the sample surface using a $20 \times$ microscope objective $(N A \approx 0.35)$. The actual writing speed, achieved with a highprecision positioning system (Aerotech) is $100 \mathrm{~mm} / \mathrm{min}$ at a pulse energy of $200 \mathrm{~nJ}$ and a repetition rate of $100 \mathrm{kHz}$ (Coherent Mira/Reg A). Such waveguides exhibit low propagation losses $(<0.3 \mathrm{~dB} / \mathrm{cm})$ and almost vanishing birefringence $\left(\Delta n_{H, V} \approx 10^{-6}\right)$. With a supported mode field diameter of $12 \mu \mathrm{m} \times 15 \mu \mathrm{m}$, the coupling losses of $3 \mathrm{~dB}$ are obtained with standard single mode fibers.

\section{Photon-pair generation and measurement}

We generate photon-pairs at $\lambda=815 \mathrm{~nm}$ using a standard type-I spontaneous parametric down-conversion source with a visibility of $93 \%$. A BiBO crystal is pumped by a $100-\mathrm{mW}, 407.5-\mathrm{nm}$ laser diode producing horizontally polarized photon-pairs, which are collected by polarization maintaining fibers. Commercial V-groove fiber arrays were used to couple the photons into the chip as well as collecting them at the output facet from the individual waveguides. We used high-NA multi-mode fibers in order to feed the photons to the respective avalanche photo-diodes, ensuring low coupling losses at the output side of the chip. From the data of the photo-diodes, the photon probability distribution at the output, as well as the inter-channel correlations, were computed using a correlation device (Becker-Hickl) and standard computer programs (LabView for the data acquisition and MatLab for the data processing). The photonic chip was fabricated such that the waveguide spacing at the input and output facets matched the standard fiber array spacing of $127 \mu \mathrm{m}$. The typical two-photon coincidence rates after propagating through the chip were approximately $30 \mathrm{~Hz}$, and an integration time of around $20 \mathrm{~s}$ was used for coincidence measurements.

\section{Algorithmic reconstruction}

The algorithm for reconstructing the density matrix from a measured set of correlations uses the simultaneous perturbation SPSA. ${ }^{34}$ We follow an optimization process very similar to, ${ }^{17}$ except we adapt it to work with mixed quantum states. This is achieved by defining the density matrix as $\hat{\rho}(t)=\hat{T}^{\dagger}(t) \hat{T}(t) / \operatorname{Tr}\left\{\hat{T}^{\dagger}(t) \hat{T}(t)\right\}$, as in Eq. (4.5) of ref. ${ }^{8}$. Here $T(t)$ is an upper triangular matrix with the elements in the triangle specified by the vector $t$ $=\left[t_{1}, t_{2} \ldots t_{n}\right]$, and the number of elements in $t$ is set to be equal to the number of free parameters in the density matrix. Then the algorithm proceeds optimizing $t$, rather than $\psi$ as in previous approaches for pure quantum states. We determine the reconstruction accuracy using the "experimentally measured" device transfer function. The recovery of 500 random density matrices is simulated, each for 1500 iterations of the algorithm. The reconstruction fidelity, the average fidelity between the true density matrix and the algorithm's final guess, is found to be $99.67 \%$.

For reconstruction using experimentally measured data there will naturally be errors in the amplitude of the measured correlation elements, in particular due to shot noise. This will cause the measured correlations to deviate so that they don't perfectly match the correlations that would be produced by a physical density matrix. Since the system is mathematically over-determined, i.e., we measure more correlations than unknown elements in the density matrix, not all combinations of correlations would correspond to a physical density matrix. Considering random noise, we can then estimate the error of the reconstructed state by how much the measured correlations deviate from the correlations of the closest physical density matrix, $\Gamma_{\text {rec }}$, found by the reconstruction algorithm. We perform such analysis for the split state as shown in Fig. 3a, and find that the average statistical fidelity between the reconstructed correlations $\Gamma_{\text {rec }}$ and the measured correlations $\Gamma_{\text {meas }}$ is $99.71 \%$. To ensure that the calculated statistical fidelity accurately represents the noise in the system, the measured correlations were recorded with a moving average of $13 \mathrm{~s}$ over a total time of $60 \mathrm{~s}$ and the average statistical fidelity was calculated for each $13 \mathrm{~s}$ window, then the mean value was taken. The statistical fidelity was 
calculated according to $F=\left\langle\sum_{i} \sqrt{\Gamma_{\text {meas }}^{(i)} \Gamma_{\text {rec }}^{(i)}}\right\rangle\left(\right.$ as in $\left.^{13}\right)$, where $i$ is the index of different correlation matrix elements, and brackets denote statistical averaging. Note that the correlation matrices are normalized to sum to 1 since they are probabilities. For this normalization the diagonal correlation elements (corresponding to detection of two photons at the same detector) are set to zero since we do not use photon number resolved detection, and thus do not measure these elements.

Data availability

The data that support the findings of this study are available from the corresponding author upon reasonable request.

\section{ACKNOWLEDGEMENTS}

We acknowledge support by the Australian Research Council (ARC) (DP130100135, DP160100619 and DE180100070); Erasmus Mundus (NANOPHI 2013 5659/002-001); Alexander von Humboldt-Stiftung; Australia-Germany Joint Research Co-operation Scheme of Universities Australia; German Academic Exchange Service (project 57376641), and the Deutsche Forschungsgemeinschaft (grants SZ 276/12-1 and BL 574/13-1).

\section{AUTHOR CONTRIBUTIONS}

J.G.T, A.S.S, and A.A.S developed the theoretical concept. J.G.T, M.G, R.H, and A.S designed and fabricated the photonic chip. J.G.T, M.G, and R.H preformed the experiments. All authors co-wrote the manuscript.

\section{ADDITIONAL INFORMATION}

Supplementary information accompanies the paper on the npj Quantum Information website (https://doi.org/10.1038/s41534-018-0063-5).

Competing interests: The authors declare no competing financial interests.

Publisher's note: Springer Nature remains neutral with regard to jurisdictional claims in published maps and institutional affiliations.

\section{REFERENCES}

1. Barends, R. et al. Superconducting quantum circuits at the surface code threshold for fault tolerance. Nature 508, 500-503 (2014).

2. Wang, X. L. et al. Experimental ten-photon entanglement. Phys. Rev. Lett. 117, 210502 (2016).

3. Monz, T. et al. 14-qubit entanglement: creation and coherence. Phys. Rev. Lett. 106, 130506 (2011).

4. Nielsen, M. A. \& Chuang, I. L. Quantum Computation and Quantum Information. 10th anniv. edn, (Cambridge University Press, Cambridge, 2011).

5. Altepeter, J. B., Jeffrey, E. R. \& Kwiat, P. G. Photonic state tomography. Adv. Atom. Mol. Opt. Phys. 52, 105-159 (2005).

6. Banaszek, K., Cramer, M. \& Gross, D. Focus on quantum tomography. New J. Phys. 15, 125020 (2013).

7. Vogel, K. \& Risken, H. Determination of quasiprobability distributions in terms of probability-distributions for the rotated quadrature phase. Phys. Rev. A 40, 2847-2849 (1989).

8. James, D. F. V., Kwiat, P. G., Munro, W. J. \& White, A. G. Measurement of qubits. Phys. Rev. A 64, 052312 (2001).

9. Haffner, H. et al. Scalable multiparticle entanglement of trapped ions. Nature $\mathbf{4 3 8}$, 643-646 (2005).

10. Leibfried, D. et al. Creation of a six-atom 'Schrödinger cat' state. Nature 438, 639-642 (2005).

11. Lvovsky, A. I. \& Raymer, M. G. Continuous-variable optical quantum-state tomography. Rev. Mod. Phys. 81, 299-332 (2009).

12. Bayraktar, O., Swillo, M., Canalias, C. \& Bjork, G. Quantum-polarization state tomography. Phys. Rev. A 94, 020105 (2016).

13. Shadbolt, P. J. et al. Generating, manipulating and measuring entanglement and mixture with a reconfigurable photonic circuit. Nat. Photonics 6, 45-49 (2012).
14. Gross, D., Liu, Y. K., Flammia, S. T., Becker, S. \& Eisert, J. Quantum state tomography via compressed sensing. Phys. Rev. Lett. 105, 150401 (2010).

15. Oren, D., Shechtman, Y., Mutzafi, M., Eldar, Y. C. \& Segev, M. Sparsity-based recovery of three-photon quantum states from two-fold correlations. Optica 3, 226-232 (2016).

16. Cramer, M. et al. Efficient quantum state tomography. Nat. Commun. 1, 149 (2010).

17. Ferrie, C. Self-guided quantum tomography. Phys. Rev. Lett. 113, 190404 (2014).

18. Chapman, R. J., Ferrie, C. \& Peruzzo, A. Experimental demonstration of self-guided quantum tomography. Phys. Rev. Lett. 117, 040402 (2016).

19. Qi, B. et al. Adaptive quantum state tomography via linear regression estimation: theory and two-qubit experiment. npj Quant. Inform. 3, 19 (2017).

20. Miranowicz, A. et al. Optimal two-qubit tomography based on local and global measurements: Maximal robustness against errors as described by condition numbers. Phys. Rev. A 90, 062123 (2014).

21. Lundeen, J. S., Sutherland, B., Patel, A., Stewart, C. \& Bamber, C. Direct measurement of the quantum wavefunction. Nature 474, 188-191 (2011).

22. Bolduc, E., Gariepy, G. \& Leach, J. Direct measurement of large-scale quantum states via expectation values of non-Hermitian matrices. Nat. Commun. 7, 10439 (2016).

23. Thekkadath, G. S. et al. Direct measurement of the density matrix of a quantum system. Phys. Rev. Lett. 117, 120401 (2016).

24. Heilmann, R. et al. Harnessing click detectors for the genuine characterization of light states. Sci. Rep. 6, 19489 (2016).

25. Titchener, J. G., Solntsev, A. S. \& Sukhorukov, A. A. Two-photon tomography using on-chip quantum walks. Opt. Lett. 41, 4079-4082 (2016).

26. Oren, D., Mutzafi, M., Eldar, Y. C. \& Segev, M. Quantum state tomography with a single measurement setup. Optica 4, 993-999 (2017).

27. Meany, T. et al. Laser written circuits for quantum photonics. Laser Photon. Rev. 9, 363-384 (2015).

28. Heilmann, R., Grafe, M., Nolte, S. \& Szameit, A. A novel integrated quantum circuit for high-order W-state generation and its highly precise characterization. Sci. Bull. 60, 96-100 (2015).

29. Foreman, M. R., Favaro, A. \& Aiello, A. Optimal frames for polarization state reconstruction. Phys. Rev. Lett. 115, 263901 (2015).

30. Hong, C. K., Ou, Z. Y. \& Mandel, L. Measurement of subpicosecond time intervals between two photons by interference. Phys. Rev. Lett. 59, 2044-2046 (1987).

31. Spring, J. B. et al. Chip-based array of near-identical, pure, heralded single-photon sources. Optica 4, 90-96 (2017).

32. Mahrlein, S., von Zanthier, J. \& Agarwal, G. S. Complete three photon Hong-OuMandel interference at a three port device. Opt. Express 23, 15833-15847 (2015).

33. Ou, Z. Y., Zou, X. Y., Wang, L. J. \& Mandel, L. Experiment on nonclassical fourthorder interference. Phys. Rev. A 42, 2957-2965 (1990).

34. Spall, J. C. Multivariate stochastic-approximation using a simultaneous perturbation gradient approximation. IEEE Trans. Autom. Control 37, 332-341 (1992).

35. Hamel, D. R. et al. Direct generation of three-photon polarization entanglement. Nat. Photonics 8, 801-807 (2014).

36. White, A. G., James, D. F. V., Eberhard, P. H. \& Kwiat, P. G. Nonmaximally entangled states: Production, characterization, and utilization. Phys. Rev. Lett. 83, 3103-3107 (1999).

37. Najafi, F. et al. On-chip detection of non-classical light by scalable integration of single-photon detectors. Nat. Commun. 6, 5873 (2015).

(c) Open Access This article is licensed under a Creative Commons Attribution 4.0 International License, which permits use, sharing, adaptation, distribution and reproduction in any medium or format, as long as you give appropriate credit to the original author(s) and the source, provide a link to the Creative Commons license, and indicate if changes were made. The images or other third party material in this article are included in the article's Creative Commons license, unless indicated otherwise in a credit line to the material. If material is not included in the article's Creative Commons license and your intended use is not permitted by statutory regulation or exceeds the permitted use, you will need to obtain permission directly from the copyright holder. To view a copy of this license, visit http://creativecommons. org/licenses/by/4.0/.

(c) The Author(s) 2018 